\section{Akute Attacke behoben - aber die Gicht schwelt weiter}

Abb.: Doppelkontur im Fuß-Ultraschall bei einem Gichtpatienten

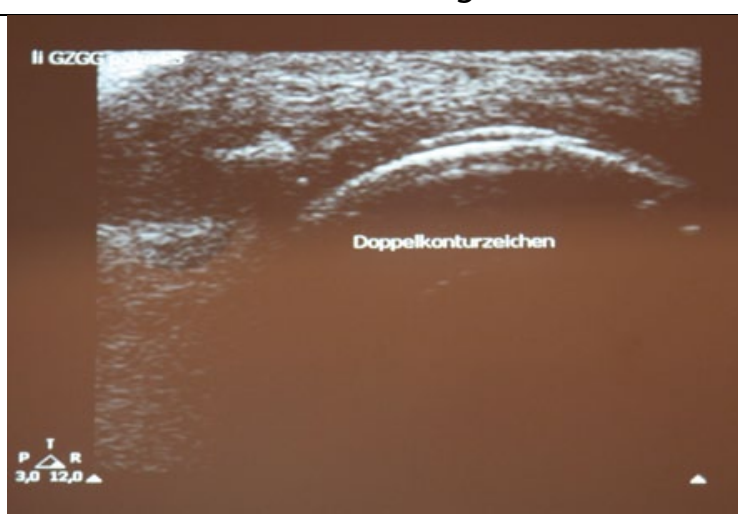

Halswirbelsäule können ihre Ursache in einer unerkannten Hyperurikämie haben, sagte die Expertin und riet, bei unklaren Gelenkbeschwerden auch den Harnsäurespiegel zu bestimmen. Als therapeutischen Zielwert im Fall einer Hyperurikämie nannte Reuss-Borst eine Harnsäure von $6,0 \mathrm{mg} / \mathrm{dl}$, um die bereits abgelagerten Harnsäurekristalle wieder in Lösung zu bekommen.

Gerne vergessen wird, dass die Harnsäuremessung im akuten Gichtanfall wenig aussa- gekräftig ist, da dann oft eine Normourikämie besteht. Eine realistische Einschätzung gibt die Messung erst zwei Wochen nach der akuten Attacke. Der Kristallnachweis gilt weiter als diagnostischer Goldstandard, erfolgt heute aber seltener nach Gelenkpunktion in der Gelenkflüssigkeit. Stattdessen gewinnt der Ultraschall in der Hand geübter Ärzte immer mehr an Bedeutung: das Doppelkonturzeichen gilt als pathognomonisch für Kristallablagerungen (Abb.).

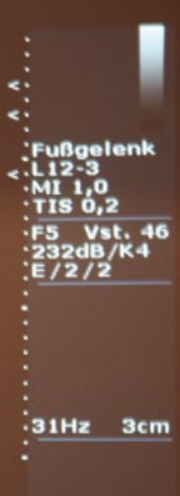

\section{Immunsystem setzt den Knochen von RA-Patienten zu}

— Unter dem Titel „Immunobone“ berichtete Prof. Georg Schett, Erlangen, über die Kommunikation zwischen Knochen und Immunsystem sowie über die Prozesse, die dem Knochenabbau bei entzündlich rheumatischen Erkrankungen zugrunde liegen. Sein plastisches Beispiel: So, wie ein Stein, der kontinuierlich von Wasser umspült wird, langsam runder und kleiner wird, ergeht es auch dem Knochen: Er wird von einem "Meer" aus Zytokinen umflutet, die ihn allmählich resorbieren. Die beiden beteiligten Pathomechanismen sind die Entzündung und die Autoimmunität.

Erwartungsgemäß ist der knöcherne Schaden umso größer, je höher die Zytokinspiegel sind. Allerdings ist Zytokin nicht gleich
Zytokin. Während Tumor-Nekrose-Faktor alpha (TNF a) und die Interleukine (IL) 1 und 6 die Osteoklastogenese massiv fördern, gilt dies nicht für Interferon (IFN). Das erklärt auch, warum sich das Ausmaß der Knochenresorption bei rheumatischen Erkrankungen unterscheidet. Während sie bei der TNF-aund IL-1-getriebenen RA stark ausgeprägt ist, gilt dies weniger für den IFN-betonten systemischen Lupus erythematosus (SLE); der in geringerem Maß knochenerosiv verläuft. Bei der äußerst erosiven Gicht ist die ossäre Resorption primär IL-1-vermittelt. Aufgrund der Bedeutung von TNF a für die Erosion wird auch klar, warum unter TNF-aBlockade eine gewisse Heilungstendenz am Knochen zu beobachten ist.

\title{
Stammzelltransplantation bei Systemsklerose
}

- Bisher gibt es nur wenige und nicht ausreichend effektive Therapieoptionen für die destruktive und mit hoher Mortalität verknüpfte progressive Systemsklerose (SSC). Möglich ist die Gabe von Methotrexat, Azathioprin, Mycophenolat, bei SSC mit pulmonalen Befall wird auch Cyclophosphamid eingesetzt, das häufig ebenfalls gering oder nicht anhaltend wirkt, wie Dr. Jörg Henes, Tübingen, betonte. Daher habe die symptomatische Therapie von Begleiterkrankungen der SSC einen hohen Stellen- wert. Für Rituximab und Imatinib gebe es erste vielversprechende Fallbeispiele.

Wegen der unbefriedigenden Therapiesituation plädierte Henes für weitere Studien zur autologen Stammzelltransplantation. Denn bei Patienten mit therapierefraktärer SSC sei dies inzwischen das am besten untersuchte und wirksamste Verfahren, wie unter anderem die im Sommer beim EULARKongress in Berlin vorgestellte Phase-IIIStudie ASTIS (Autologous Stem cell Transplantation International Scleroderma trial)
Bislang ging man eher davon aus, dass bei rheumatischen Erkrankungen die Entzündung der Autoimmunität vorausgeht. Dies scheint nicht zuzutreffen, wie neue Untersuchungen zu den Antikörpern gegen citrullinierte Proteine/Peptide (ACPA) zeigen. Als entzündungsunabhängiger Risikofaktor gelten sie nicht nur als Prädiktor für einen schwereren Verlauf der RA, sondern auch für einen stärkeren Knochenverlust. Denn ACPA induzieren die Differenzierung der Osteoklasten. Sie sind also kein Epiphänomen, sondern tatsächlich ein Risikofaktor und zwar einer, der bereits zehn Jahre vor Erkrankungsbeginn anzutreffen ist. So überrascht es nicht, dass ACPA-positive RA-Patienten zum Zeitpunkt der Diagnose oft bereits eine Osteopenie oder gar knöcherne Schäden aufweisen.

wk

zeigen konnte. In der Studie wurde die Effektivität der Stammzelltransplantation nach einer Hochdosischemotherapie mit einer Cyclophosphamid-Stoßtherapie verglichen. Die Stammzelltransplantation erwies sich im längerfristigen Verlauf sowohl hinsichtlich der Remissionsrate als auch der Mortalität als überlegen. Die 100-TageMortalität war dagegen höher. Damit sei die Frage der Effektivität geklärt, meinte Henes. In weiteren Studien müsse der Therapieansatz nun hinsichtlich der optimalen Patientenselektion und der Reduktion der Toxizität optimiert werden. 